\title{
New methodologies for the analysis and synthesis of oscillator circuits
}

\author{
Almudena Suárez, Mabel Pontón, Sergio Sancho, Franco Ramírez \\ Department of Communications Engineering \\ Santander, Spain \\ almudena.suarez@unican.es; mabel.ponton@unican.es; sanchosm@unican.es; ramirezf@unican.es
}

\begin{abstract}
Advances in the analysis and synthesis of oscillator circuits, using harmonic balance (HB), are presented. They rely on the use of auxiliary generators, which can be introduced into the $\mathrm{HB}$ software to impose mathematical conditions or to extract a realistic oscillator model. In particular, a bifurcation-detection technique, for the accurate design of dual-frequency oscillators, and a semi-analytical function, for the prediction of oscillation transients, are described. In dual-frequency oscillators, each oscillation must be the only stable solution in a certain parameter interval. This is ensured through the calculation of two distinct primaryHopf bifurcation loci, which should give rise to disjoint parameter regions. Conditions for the physical observability of concurrent oscillations are also given. With respect to the transient prediction, both the linear and nonlinear stages are considered. The analysis is based on the derivation of outer-tier semi analytical equation, from which a growth rate function is identified, which, unlike ordinary simulations, is not constrained to particular initial values. The methods have been applied to two FET-based oscillator circuits that have been manufactured and measured, obtaining good agreement with the simulation results.
\end{abstract}

\section{Keywords-Oscillator, two-tier methodology, transient}

\section{INTRODUCTION}

In the last years, numerous efforts [1]-[6] have been devoted to the development of novel analysis and synthesis tools for oscillator circuits, intended to be applied in combination with the harmonic-balance (HB) method [7][8]. In fact, $\mathrm{HB}$ suffers from a number of shortcomings when dealing with autonomous circuits, mostly due to the fact that it is insensitive to the stability properties of the solution, generated and extinguished through bifurcation phenomena [8]. Two main strategies have been proposed to overcome the problem, based on the use of auxiliary generators (AGs). In the first one, the $\mathrm{AG}$ is introduced into the HB software to maintain the oscillation through a complex topology, as in N-push [1] and rotary travelling wave oscillators [2], or detect bifurcation points [3]. The second strategy is the use of the AG to extract a realistic model of a core oscillator, later introduced in a semianalytical formulation of this oscillator under certain (more complex) operation conditions [4],[5]. This has been applied for the analysis and design of frequency dividers and coupled-oscillator systems [4], among other. The procedure combines the accuracy of commercial software in terms of passive/active modelling (inner tier) with a dedicated formulation/analysis (outer tier) of the circuit or system.

Here two recent advances in the complementary analyses methods are presented. The first one addresses the efficient design of dual-frequency oscillators [5], of interest in compact and low-consumption reconfigurable communication systems [9]-[10]. However, the dualfrequency oscillators suffer from a likely coexistence of stable solutions, and, thus, uncertainty in the circuit operation. Here a bifurcation-analysis technique is provided to accurately determine the parameter values leading to each oscillation. The second methodology addresses the prediction of the oscillator transient [6], highly relevant in mobile communications [11]-[13], where a fast oscillation start-up reduces the power consumption. A regular oscillator transient is also essential in envelope-modulation systems and radar. Here, a growth-rate function will be derived, enabling a global prediction of the circuit transient response, not constrained to particular initial values, as in timedomain integration.

\section{DUAL-FREQUENCY OSCILLATOR}

A dual-frequency oscillator can be based on two crosscoupled transistors, to obtain negative resistance, as well as a double resonator, acting as a load element (Fig. 1). Oscillation at the lower frequency $\omega_{d}$, higher frequency $\omega_{h}$ or both should be achieved under the variation of certain circuit parameters. Here these parameters are the varactorbias voltage $V_{v a r}$ and the drain-bias voltage $V_{D D}$. The initial goal is to determine the parameter region in which the circuit oscillates at each of the two frequencies. At steadystate oscillations, the current-to-voltage ratio is equal to zero at all the circuit nodes (oscillation condition). On the other hand, ordinary oscillations are generated/extinguished in a primary Hopf bifurcation [14], where a pair of complexconjugate poles crosses the imaginary axis, giving rise to a qualitative stability change of the dc solution. At the primary Hopf bifurcations, the oscillation condition is fulfilled with zero amplitude.

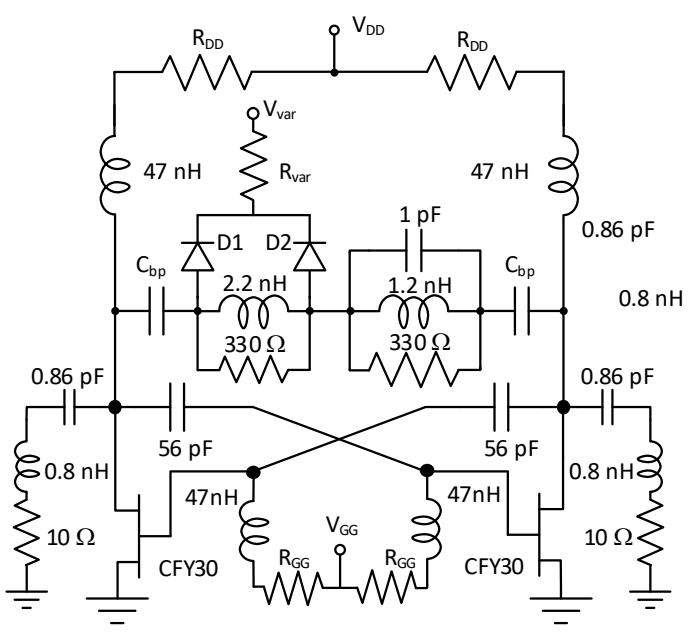

Fig. 1. Dual-frequency oscillator at $f_{d}=900 \mathrm{MHz}$ and $f_{h}=2.5 \mathrm{GHz}$. 
The parameter boundaries for oscillation at $\omega_{d}$ and $\omega_{h}$ can be obtained with the aid of an AG [1]-[6], which is an independent voltage source at the frequency $\omega_{A G}$ in series with an ideal bandpass filter at the same frequency. In the circuit in Fig. 1, this AG will be connected in parallel across the two transistors. The primary Hopf bifurcation at $\omega_{d}\left(\omega_{h}\right)$ is obtained by alternatively doing $\omega_{A G}=\omega_{d}\left(\omega_{A G}=\omega_{d}\right)$ and setting the AG amplitude to small signal $A_{A G}=\varepsilon$. Each of the two primary-Hopf bifurcation loci is given by:

$$
Y_{A G}\left(V_{D D}, V_{v a r}, \omega_{A G}\right)=0
$$

where $Y_{A G}$ is the ratio between the AG current. In the circuit of Fig. $1, f_{d} \cong 900 \mathrm{MHz}$ and $f_{h} \cong 2.5 \mathrm{GHz}$. For $V_{G S}=-0.7 \mathrm{~V}$, the resulting Hopf loci are shown in Fig. 2(a). For too low $V_{D D}$, the $d c$ solution is stable (non oscillatory) for all the $V_{v a r}$ values. The intersection of the two Hopf loci at the point $\mathrm{X}_{2}$ splits the $V_{\text {var }}$ interval into two subintervals. At the lower (upper) subinterval, the oscillation at $\omega_{d}\left(\omega_{h}\right)$ arises from a stable dc regime. Only oscillations generated from a stable $d c$ regime are originally stable, or become stable just after a turning point. Thus, it will be possible to obtain the two oscillations independently. Fig. 3(a) and (b) respectively show measurements for $V_{v a r}$ above and below $\mathrm{X}_{2}$, when varying $V_{D D}$. Other loci in Fig. 2(a) correspond to secondary Hopf bifurcations, arising from periodic regime.

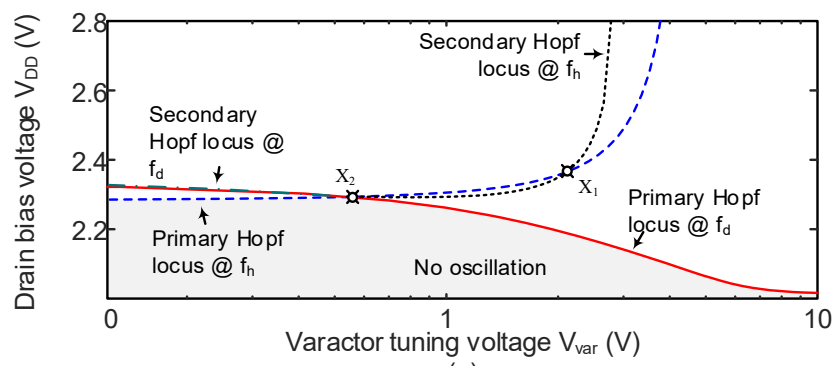

(a)

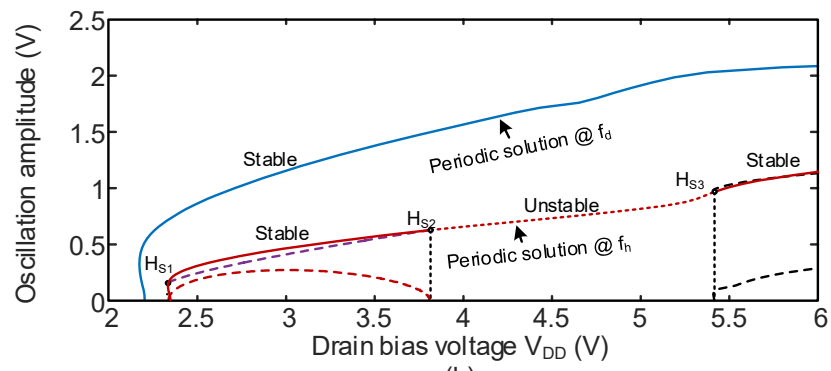

(b)

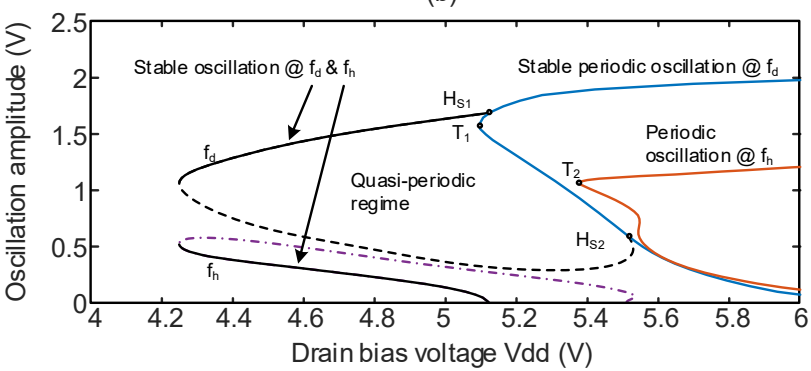

(c)

Fig. 2. Dual-frequency oscillator with $V_{\mathrm{var}}=1.9 \mathrm{~V}$. (a) Primary and secondary Hopf-bifurcation loci in the plane $\left(V_{D D}, V_{\text {var }}\right)$. (b) Bifurcation diagram vs. $V_{D D}$ for $V_{G S}=-0.7 \mathrm{~V}$ and $V_{\text {var }}=1.9 \mathrm{~V}$. (c) For $V_{G S}=-1.4 \mathrm{~V}$ and $V_{\text {var }}=3.5 \mathrm{~V}$.
The steady-state oscillation curves are obtained by solving: $Y_{A G}\left(\eta, A_{A G}, \omega_{A G}\right)=0$, where the parameter $\eta$ is either $V_{\text {var }}$ or $V_{D D}$. Fig. 2(b) shows the solution diagram versus $V_{D D}$ for $V_{\text {var }}=1.9 \mathrm{~V}$. The upper section of the periodic solution curve at $f_{d}$ is stable. The periodic solution curve at $f_{h}$ is originally unstable, due to the presence of a pair of complex-conjugate poles (with frequency in the order of $f_{d}$ ) on the right-hand side of the complex plane (RHS). These poles cross the imaginary axis to the left side (LHS) at $\mathrm{H}_{\mathrm{s} 1}$, which leads to the stabilization of the periodic solution at $f_{h}$. They cross again to RHS at $\mathrm{H}_{\mathrm{S} 2}$, from which the solution at $f_{h}$ becomes unstable. It becomes stable again at $\mathrm{H}_{\mathrm{s} 3}$. At $\mathrm{H}_{\mathrm{s} 1}$ and $\mathrm{H}_{\mathrm{s} 3}$, a quasi-periodic regime is generated, at the two concurrent oscillations at $\omega_{d}$ and $\omega_{h}$. Only quasiperiodic solutions arising from a stable periodic solution are initially stable, so all the quasi-periodic solutions in Fig. 2(b) are unstable. These solutions are calculated introducing two AGs into the circuit, at $\omega_{A G 1}, \omega_{A G 2}$, and solving the outer-tier system:

$$
\begin{aligned}
& Y_{A G, 1}\left(A_{A G 1}, \omega_{A G 1}, A_{A G 2}, \omega_{A G 2}\right)=0 \\
& Y_{A G, 2}\left(A_{A G 1}, \omega_{A G 1}, A_{A G 2}, \omega_{A G 2}\right)=0
\end{aligned}
$$

In turn, the secondary Hopf bifurcations are obtained by setting one of the two amplitudes to small signal $A_{A G i}=\varepsilon$, where $i=1,2$, and solving for the analysis parameter $\eta$. Secondary Hopf-bifurcation loci are also traced in Fig. 2(a), in terms of $V_{D D}$ and $V_{v a r}$. Due to the turning points of the periodic curves, secondary Hopf bifurcations appear before the primary Hopf ones in the interval $\left(\mathrm{X}_{1}, \mathrm{X}_{2}\right)$. At $V_{G S}=$ $1.4 \mathrm{~V}$ [Fig. 2(c)], when reducing $V_{D D}$ from high value the oscillation at $f_{d}$ is stable and the Hopf bifurcation $\mathrm{H}_{\mathrm{s} 1}$ takes place from a stable periodic solution, leading to a stable quasiperiodic solution with two concurrent oscillations at $f_{d}$ and $f_{h}$. The experimental spectrum is shown in Fig. 3(c). The two independent oscillations are able to synchronize, as demonstrated in [14] [Fig. 3(d)]. Fig. 4 presents the measured phase noise of the oscillation at $f_{d}, f_{h}$ and the synchronized-concurrent oscillation at both $f_{d}$ and $f_{h}$, with lower phase noise than the individual oscillations.
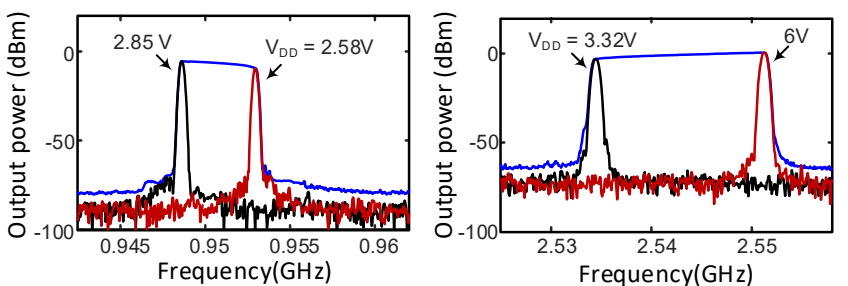

(a)

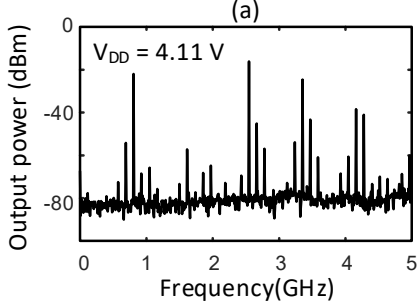

(b)

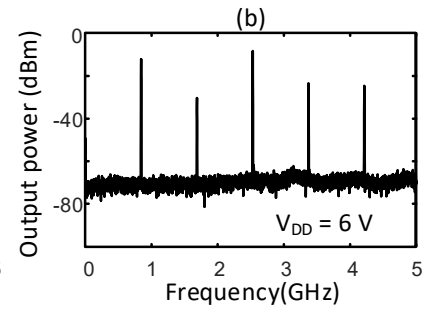

(d)

Fig. 3. Experimental measurements below and above the point $\mathrm{X}_{2}$ in Fig. 2(a). (a) Mode at $f_{d}$. (b) Mode at $f_{h}$. (c) Concurrent oscillation at $f_{d}$ and $f_{h}$. (d) Synchronized-concurrent oscillation. 


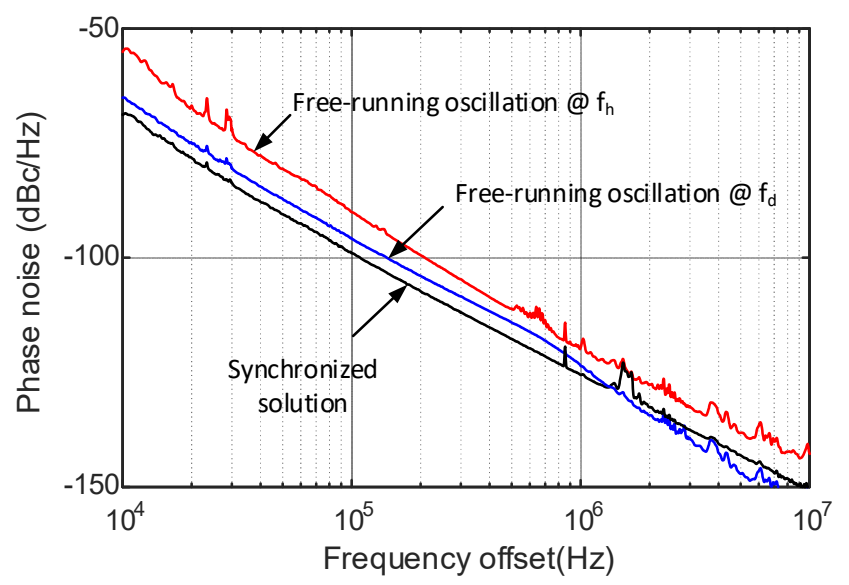

Fig. 4. Measured phase noise of the dual-frequency oscillator.

\section{OSCILLATOR TRANSIENT DYNAMICS}

The aim of the new simulation technique is to analyze the whole transient trajectory, from the initial value to the steady-state oscillation. For illustration, it will be applied to the oscillator of Fig. 5. The Implicit Function Theorem (IFT) can be applied [14], in combination with HB, to obtain an outer-tier equation, describing the free-running oscillator circuit. This can be referred to the first harmonic of the voltage and current at a given observation node $q$ :

$$
I_{1}\left(X_{1}, \omega\right)=Y(V, \omega) V e^{j \phi}, \quad X_{1}=V e^{j \phi}
$$

where $Y(V, \omega), X_{1}$ are the oscillator admittance function and the first harmonic component of the voltage at the $q$ node, respectively. During the transient state, $X_{1}(t)$ is time varying. Then, following the method described in [14]-[15], the static equation (3) is translated to the envelope domain to model the dynamics of $X_{1}(t)$ :

$$
\begin{aligned}
& Y(t) V(t) e^{j \phi(t)}+Y_{\omega}(t)\left[\dot{\phi}_{1}(t) V(t)-j \dot{V}(t)\right] e^{j \phi(t)}=0, \\
& Y(t) \equiv Y\left[V(t), \omega_{o}\right]
\end{aligned}
$$

where $\omega_{o}$ is the free-running frequency and the subindex $\omega$ indicates differentiation with respect to the frequency. Solving the complex equation (4) for $V(t)$ one obtains:

$$
\dot{V}(t)=g[V(t)], \quad g[V(t)] \equiv \frac{Y^{i}(t) Y_{\omega}^{r}(t)-Y^{r}(t) Y_{\omega}^{i}(t)}{\left|Y_{\omega}(t)\right|^{2}} V(t)
$$

where the superscripts $r, i$ indicate real and imaginary parts. The admittance functions $Y\left(V, \omega_{o}\right), Y_{\omega}\left(V, \omega_{o}\right)$ can be calculated through circuit-level HB simulations as described in [10]. The admittance function $Y\left(V, \omega_{o}\right)$ is calculated through an amplitude sweep, doing $Y\left(V, \omega_{o}\right)=I_{A G}\left(V_{A G}, \omega_{A G}\right) / V_{A G}$, for $V_{A G}=V, \omega_{A G}=\omega_{o}$. The derivative $Y_{\omega}\left(V, \omega_{o}\right)$ is calculated through finite differences with $\omega_{A G}=\omega_{o} \pm \Delta \omega$. The function $g(V)$ is able to predict the trajectory without numerical integration, and starting from any initial value of $V$. This has been validated through comparison with circuit-level envelope-transient simulations. In Fig. 6, the oscillator transient trajectory has been analyzed in the phase-space $(V, \dot{V})$ for two values of the varactor bias $V_{\text {var. }}$. The trajectories obtained through circuit-level envelope-transient simulation [16] start close to the dc solution $(V \approx 0)$ and end at the steady state value $V=V_{o}\left(V_{\text {var }}\right)$, for which $\dot{V}=g\left(V_{o}\right)=0$. The transient becomes slower as $V_{v a r}$ is increased, in agreement with the decrease of $g(V)$ with this parameter. This has been confirmed by the measurements provided in Table I.

TABLE I

MEASURED StABILIZATION TIME VS. VARACTOR BIAS

\begin{tabular}{|c|c|}
\hline$V_{\text {var }}(\mathrm{V})$ & Stabilization time $(\mu \mathrm{s})$ \\
\hline 1.5 & 3 \\
\hline 2 & 33 \\
\hline 2.5 & 109 \\
\hline
\end{tabular}

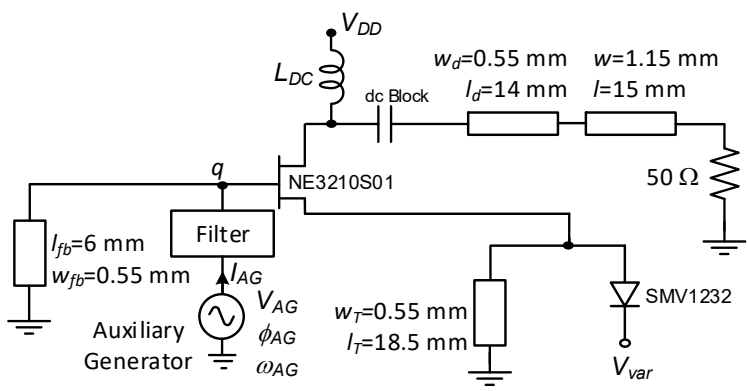

Fig. 5. Schematic of the FET oscillator at $5 \mathrm{GHz}$ with an AG.

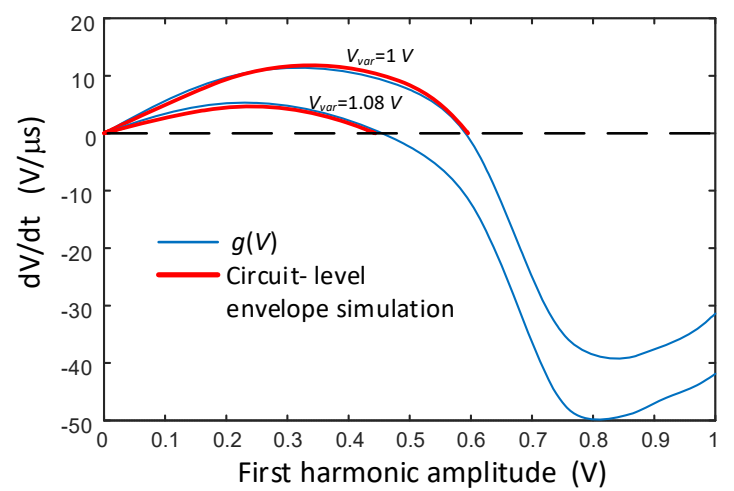

Fig. 6. Phase-space trajectories for the values $V_{v a r}=1$ and $V_{\text {var }}=1.08 \mathrm{~V}$.

\section{REFERENCES}

[1] F. Ramírez, M. Pontón, S. Sancho, A. Suárez, "Stability analysis of oscillation modes in quadruple-push and Rucker's oscillators," IEEE Trans. Microw. Theory Techn., vol. 56, no. 11, pp. 2648-2661, 2008.

[2] M. Pontón, A. Suárez, J.S. Kenney, "Rotary Traveling-Wave Oscillator With Differential Nonlinear Transmission Lines," IEEE Trans. Microw. Theory Techn., vol. 62, no. 5, pp. 1149 - 1161, 2014.

[3] A. Suárez, E. Fernández, F. Ramírez, S. Sancho, "Stability and bifurcation analysis of self-oscillating quasi-periodic regimes," IEEE Trans. Microw. Theory Techn., vol. 60, no. 3, pp. 528-541, 2012.

[4] A. Suárez, F. Ramírez, S. Sancho, "Stability and Noise Analysis of Coupled-Oscillator Systems," IEEE Trans. Microw. Theory Techn, vol. 59, no. 4, pp. 1032-1046, 2011.

[5] F. Ramírez, S. Sancho, A. Suárez, "Oscillation Modes in FreeRunning Oscillators Loaded with Multi-Resonant Networks," IEEE Trans. Microw. Theory Techn., vol. 64, no. 12, pp. 1-16, Dec., 2016. 
[6] A. Suárez, S. Sancho, and F. Ramírez, "Growth-rate function for the nonlinear analysis of the transient dynamics of microwave oscillators," in 2016 IEEE MTT-S International Microwave Symposium (IMS), May 2016, pp. 1-4.

[7] K. Kundert. "Introduction to RF simulation and its application," Proc. IEEE Bipolar/BiCMOS Circuits Techn. Meeting, vol. 34, no. 9, pp. 67- 78, Sep., 1998.

[8] V. Rizzoli and A. Neri, "State of the art and present trends in nonlinear microwave CAD techniques," IEEE Trans. Microwave Theory Tech., vol. 36, pp. 343-356, Feb.1988.

[9] A. Goel, H. Hashemi, "Concurrent Dual-Frequency Oscillators and Phase-Locked Loops”, IEEE Trans. Microw. Theory Tech., vol. 56, no. 8, pp. 1846-1860, Aug. 2008.

[10] A. Goel, H. Hashemi, "Injection Locking in Concurrent DualFrequency Oscillators", IEEE Trans. Microw. Theory Tech., vol. 56, no. 8, pp. 1834-1845, Aug. 2008.

[11] P. Gamand and V. Pauker, "Starting phenomenon in negative FET oscillators," Electronics Letters, vol. 24, no. 15, pp. 911-913, July 1988.

[12] B. Voigt, et al, "A new approach to determine the start-up time of sinusoidal oscillators", 37th Midwest Symposium on Circuits and Systems, vol. 2, pp. 1119-1122, Aug. 1994.

[13] Y. Tsuzuki, T. Adachi and J. Zhang, "Formulation of nonlinear negative resistance for calculation of start-up characteristics of crystal oscillators", Frequency Control Symposium, 1996. 50th., Proc. of the 1996 IEEE International, pp. 710-713, June 1996.

[14] A. Suarez, Analysis and Design of Autonomous Microwave Circuits. Wiley, 2009.

[15] K. Kurokawa, "Some basic characteristics of broadband negative resistance oscillator circuits," Bell Syst. Tech. J., vol. 48, pp. 19371955, July-Aug. 1969

[16] E. Ngoya, J. Rousset, D. Argollo, "Rigorous RF and microwave oscillator phase noise calculation by envelope transient technique," IEEE Microwave Symposium Digest. pp.91-94 vol.1, 11-16 June 2000 . 